\title{
A Statistical Power Comparison of the Kolmogorov-Smirnov Two-Sample Test and the Wald Wolfowitz Test in Terms of Fixed Skewness and Fixed Kurtosis in Large Sample Sizes
}

\author{
Ötüken SENGER \\ Kafkas University, Kars, Turkey
}

\begin{abstract}
In this study, the statistical powers of Kolmogorov-Smirnov two-sample (KS-2) and Wald Wolfowitz (WW) tests, non-parametric tests used in testing data from two independent samples, have been compared in terms of fixed skewness and fixed kurtosis by means of Monte Carlo simulation. This comparison has been made when the ratio of variance is two as well as with equal and different sample sizes for large sample volumes. The sample used in the study is: $(25,25),(25,50),(25,75),(25,100),(50,25),(50,50),(50,75),(50,100),(75,25),(75,50),(75,75),(75$, $100),(100,25),(100,50),(100,75)$, and $(100,100)$. According to the results of the study, it has been observed that the statistical power of both tests decreases when the coefficient of kurtosis is held fixed and the coefficient of skewness is reduced while it increases when the coefficient of skewness is held fixed and the coefficient of kurtosis is reduced. When the ratio of skewness is reduced in the case of fixed kurtosis, the WW test is stronger in sample volumes $(25,25)$, $(25,50),(25,75),(25,100),(50,75)$, and $(50,100)$ while KS-2 test is stronger in other sample volumes. When the ratio of kurtosis is reduced in the case of fixed skewness, the statistical power of WW test is stronger in volume samples $(25,25),(25,75),(25,100)$, and $(75,25)$ while KS-2 test is stronger in other sample volumes.
\end{abstract}

Keywords: Kolmogorov-Smirnov Two-Sample (KS-2) test, Wald Wolfowitz (WW) test, statistical power, skewness, kurtosis

\section{Introduction}

Two-sample statistical comparison is one of the most important hypothesis tests in the field of social sciences. Parametric or non-parametric procedures are used in this comparison. It is an important detail that the population distribution of the data was considered while using those procedures. In the case where the assumptions such as normality and variance homogeneity are broken, non-parametric statistical tests are suggested. In the cases when the data are not distributed normally, it is known that the non-parametric statistical procedures are stronger than the parametric ones. If the data received from two independent samples are to be tested with non-parametric procedures, Kolmogorov Smirnov two-sample (KS-2) test and Wald Wolfowitz (WW) test are two of the probable tests to be used.

Ötüken SENGER, Ph.D., Assistant Professor, Department of Numerical Methods, Faculty of Economic and Administrative Sciences, Kafkas University.

Correspondence concerning this article should be addressed to Assist. Prof. Dr. Ötüken SENGER (Ph.D.), Department of Numerical Methods, Faculty of Economics and Administrative Sciences, Kars Kafkas University, Kars/Turkey. E-mail: otukensenger@gmail.com. 
These two tests are often in competition with each other when the researchers select a technique for data analysis. Both KS-2 test and WW test are non-parametric tests used in testing sequenced data. Both testing methods have been designed to test the null hypothesis advocating that both populations have similar distributions against the alternative hypothesis advocating both distributions are somehow different.

The main goal of this study is to compare the statistical powers of KS-2 test and WW test when the ratio of skewness is reduced in the case of fixed kurtosis and when the ratio of kurtosis is reduced in the case of fixed skewness by means of Monte Carlo simulation.

\section{The Tests Through Which the Power Comparison Is to Be Made}

\section{KS-2 Test}

The Russian mathematician Kolmogorov developed a one-sample goodness of fit test for sequenced data in 1933 (Conover, 1999). Daniel stated that the KS-2 test was developed by Smirnov but it was named as Kolmogorov because of its similarity with the Kolmogorov one-sample test (Daniel, 1990). The KS-2 test is a general and comprehensive test that may be used if the populations of the two independent samples are equal or not (Higgins, 2004).

Conover (1999) has suggested some assumptions for the KS-2 test. They are:

- Each sample has randomly been selected from the population it represents;

- The used measurement scales are at least sequenced;

- The primarily observed variable is a continuous variable;

- The two samples are independent from each other.

Conover (1999) and Sheskin (2000) defined the data as follows:

Take $S_{1}(x)$ as sample cumulative distribution function of $X_{1}, X_{2}, \ldots, X_{n 1}$.

Take $S_{2}(x)$ as sample cumulative distribution function of $Y_{1}, Y_{2}, \ldots, Y_{n 2}$.

Marascuilo and McSweeney (1977) presented a distribution-free hypothesis in order to compare if there was any general differences between two populations. According to those statisticians, the zero and alternative hypotheses are as follows:

$\mathrm{H}_{0}$ : There is no difference between the two populations; or for all $x$ 's, $\mathrm{H}_{0}: F(x)=G(x)$; from $-\infty$ to $+\infty$.

Ha: There are some differences between the two populations; or at least for one value of $x, F(x) \neq G(x)$.

According to Daniel, the test statistic for low and large sample in KS-2 test is defined by

$$
D=\max \left|S_{1}(x)-S_{2}(x)\right|
$$

The decision rule of the hypothesis is as follows:

If the observed $D$ is higher than or equal to a critical value for the $(\alpha)$ level of significance, then the null hypothesis is rejected. In this case, it is said that there is a significant difference between the two populations.

\section{WW Test}

It is a method developed in 1940 by the Romanian mathematician Wald and Polish mathematician Wolfowitz, and uses the runs approach in searching the similarity between two sets of data (Magel \& Wibowo, 1997). This test used depends on the replications of the population differences in the case when there are two samples. However, WW sequenced numbers test may also be applied to the angular data (Sprent \& Smeeton, 2001). When applying WW runs test, the null hypothesis arguing that the distribution characteristics of the two continuous populations are equal is tested (Sahai \& Khurshid, 2002). 
Daniel suggested some assumptions for the researchers who decided to apply the WW sequenced numbers test. Daniel's assumptions are as follows:

- The data are composed of two coincidences containing a number of $X_{1}, X_{2}, X_{3}, \ldots, X_{n 1}$ observations obtained from the first population and a number of $Y_{1}, Y_{2}, Y_{3}, \ldots, Y_{n 2}$ observations obtained from the second population;

- The two samples are independent from each other;

- The variable to be tested is a continuous variable (Daniel, 1990).

In the configuration of the data sequence in the light of those assumptions, the following path is followed:

- All $N=n_{1}+n_{2}$ observations are sequences from the low towards the higher and those observations are positioned in one line such as $a_{[1]} \leq a_{[2]} \leq \ldots \leq a_{[N]}$;

- Replace any observation in this line that comes from the first sample by one, and from the second sample by two;

- For the WW sequence numbers test, the $T$ test statistic is the number of changes from one to two and from two to one (Mehta \& Patel, 1996).

According to Daniel, the null and alternative hypotheses are as follows:

$\mathrm{H}_{0}: X$ 's and $Y$ 's come from two identical populations.

$\mathrm{H}_{1}$ : The population of $X$ 's and the population of $Y$ 's are different from each other (Daniel, 1990).

Please find below the formula when one wants to apply the WW sequenced numbers test by using large samples:

$$
Z=\frac{R-\left(\frac{2 n_{1} n_{2}}{n_{1}+n_{2}}+1\right)}{\sqrt{\frac{2 n_{1} n_{2}\left(2 n_{1} n_{2}-n_{1}-n_{2}\right)}{\left(n_{1}+n_{2}\right)^{2}\left(n_{1}+n_{2}-1\right)}}}
$$

where $R$ is the number of runs. In the cases when the large sample approach is in question, the test statistic to be calculated by means of this formula is compared with the values obtained from the standard normal table for the previously determined level of significance (Daniel, 1990). If the $Z$ value is lower than or equal to the table value, the $\mathrm{H}_{0}$ hypothesis is rejected at the significance level of $\alpha$.

According to Roese, equation (2) is used in the cases when the sample sizes are not equal and one of either $n_{1}$ or $n_{2}$ is higher than 20 or if the sample sizes are equal and one of either $n_{1}$ or $n_{2}$ is higher than 100 (Roese, 2011).

\section{Skewness and Kurtosis Concepts}

The skewness and kurtosis are each specified by Balakrishnan and Nevzorov (2003) and Joanest and Gill (1998). The skewness and kurtosis standards are initially developed by Pearson in 1895 (Balakrishnan \& Nevzorov, 2003). According to Pearson, the skewness is shown with equation (3):

And the kurtosis is shown with equation (4):

$$
\gamma_{1}=\frac{\beta_{3}}{\sqrt{\beta_{2}^{3}}}
$$

$$
\gamma_{2}=\frac{\beta_{4}}{\sqrt{\beta_{2}^{2}}}
$$

where: 
$\beta_{2}$ is the second central moment of the population distribution function;

$\beta_{3}$ is the third central moment of the population distribution function;

$\beta_{4}$ is the fourth central moment of the population distribution function.

To calculate the skewness and kurtosis, Algina, Olejnik, and Ocanto (1989) suggested the following formulas:

$$
\begin{gathered}
\gamma_{1}=\frac{\mu_{3}}{\sigma^{3}} \\
\gamma_{2}=\frac{\mu_{4}}{\sigma^{4}}
\end{gathered}
$$

According to Algina, Olejnik, and Ocanto (1989), $\gamma_{1}=0$ and $\gamma_{2}=3$ for a normal distribution. Balakrishnan and Nevzorov (2003) defined $\gamma_{1}>0$ as a positive skewness; $\gamma_{1}<0$ as a negative skewness; and $\gamma_{2}=3$ as a normal distribution; $\gamma_{2}<3$ as a slight kurtosis distribution; and $\gamma_{2}>3$ as a very kurtosis distribution.

\section{Monte Carlo Study}

The Monte Carlo simulation has been used in this study aiming to compare the statistical power of the KS-2 test and WW test. The Monte Carlo simulation is defined as an approach presenting an alternative to analytic mathematics to find out the sample distribution of a statistic and the evaluation of its behavior in random samples. In Monte Carlo simulation, a statistic is experimentally calculated by using random samples taken from simulated data of a known population (Mooney, 1997).

In this study, the Monte Carlo simulation has been conducted through the SAS 9.00 computer program. The RANNOR procedure in the SAS program is used to generate random numbers from a normal distribution having an average of zero and standard deviation of one. After organizing the sample code in relation to the different skewness and kurtosis values, the power simulations are shown through PROC NPAR1WAY procedure.

This study aims to compare the statistical powers of KS-2 and WW tests due to the change of the coefficient of the skewness and kurtosis in the case where the ratio of the variances is held fixed at two. For this purpose, the coefficient of kurtosis $\left(\gamma_{2}\right)$ has been taken as fixed and zero, the coefficient of skewness $\left(\gamma_{1}\right)$ is reduced from 0.75 to 0.00 and then the coefficient of skewness $\left(\gamma_{1}\right)$ has been taken as fixed and zero and the coefficient of kurtosis $\left(\gamma_{2}\right)$ has been reduced from 3.75 to -1.00 , and the changes in the statistical power of these two tests have been measured. For both the coefficient of skewness in the case of fixed kurtosis and the coefficient of kurtosis in the case of fixed skewness, the power function developed by Fleishman has been used. There are four distributions with zero coefficient of kurtosis and 20 distributions with zero coefficient of skewness in power function of Fleishman, which is used in this study.

In comparing the statistical powers of KS-2 and WW tests in both fixed skewness and kurtosis, four equal and 12 non-equal, in all 16 large sample volumes have been used. The sample volumes used in the study are $(25,25),(25,50),(25,75),(25,100),(50,25),(50,50),(50,75),(50,100),(75,25),(75,50),(75,75),(75$, $100),(100,25),(100,50),(100,75)$, and $(100,100)$.

In this study aiming to compare the statistical powers of two non-parametric tests in the case of fixed skewness and fixed kurtosis, the $\alpha$ level of significance has been taken as 0.05 . In the study, four distributions with fixed and zero coefficient of kurtosis, and 20 distributions with fixed and zero coefficient of skewness, and 16 different sample volumes have been used. However, the ratio of variances has been taken as fixed at two. 
Therefore, totally 384 different syntaxes have been written for KS-2 and WW tests and 30,000 replications have been conducted for every case.

\section{Simulation Results}

\section{Results for the Cases of Fixed Kurtosis}

The simulation has been carried out for four distributions in the case of fixed and zero coefficient of kurtosis. According to the results of the simulation, a decrease is observed in the statistical powers of both tests when the coefficient of kurtosis is taken fixed while the coefficient of skewness is reduced. The greatest decrease in statistical power is observed when the coefficient of skewness reduces from 0.75 to 0.50 . The least power decrease is observed when coefficient of skewness reduces from 0.25 to 0.00 .

When the skewness value is reduced in the case of fixed kurtosis, the WW test is stronger in sample volumes $(25,25),(25,50),(25,75),(25,100),(50,75)$, and $(50,100)$, while KS-2 test is stronger in other sample volumes. The simulation results of the case of fixed kurtosis are shown in Table 1.

Table 1

The Statistical Power Comparison of KS-2 and WW Tests When the Skewness Value Is Reduced in the Case of Fixed Kurtosis, While $\sigma_{1}: \sigma_{2}=2$ in Large Sample Volumes

\begin{tabular}{|c|c|c|c|c|c|c|c|c|c|c|}
\hline \multirow{3}{*}{$\sigma_{1}: \sigma_{2}$} & \multirow{3}{*}{$n_{1}$} & \multirow{3}{*}{$n_{2}$} & \multicolumn{8}{|c|}{ Statistical power values of KS-2 and WW tests } \\
\hline & & & \multicolumn{2}{|c|}{$\begin{array}{l}\gamma_{1}=0.75 \\
\gamma_{2}=0.00\end{array}$} & \multicolumn{2}{|c|}{$\begin{array}{l}\gamma_{1}=0.50 \\
\gamma_{2}=0.00\end{array}$} & \multicolumn{2}{|c|}{$\begin{array}{l}\gamma_{1}=0.25 \\
\gamma_{2}=0.00\end{array}$} & \multicolumn{2}{|c|}{$\begin{array}{l}\gamma_{1}=0.00 \\
\gamma_{2}=0.00\end{array}$} \\
\hline & & & KS-2 & WW & KS-2 & WW & $\mathrm{KS}-2$ & WW & KS-2 & WW \\
\hline \multirow{16}{*}{2} & 25 & 25 & 0.331 & 0.509 & 0.183 & 0.309 & 0.154 & 0.264 & 0.148 & 0.252 \\
\hline & 25 & 50 & 0.507 & 0.661 & 0.307 & 0.359 & 0.268 & 0.273 & 0.253 & 0.253 \\
\hline & 25 & 75 & 0.642 & 0.892 & 0.373 & 0.624 & 0.317 & 0.495 & 0.306 & 0.472 \\
\hline & 25 & 100 & 0.651 & 0.923 & 0.400 & 0.647 & 0.340 & 0.506 & 0.335 & 0.484 \\
\hline & 50 & 25 & 0.509 & 0.324 & 0.240 & 0.158 & 0.196 & 0.135 & 0.189 & 0.132 \\
\hline & 50 & 50 & 0.819 & 0.800 & 0.478 & 0.502 & 0.400 & 0.410 & 0.379 & 0.391 \\
\hline & 50 & 75 & 0.896 & 0.892 & 0.588 & 0.572 & 0.507 & 0.455 & 0.486 & 0.436 \\
\hline & 50 & 100 & 0.948 & 0.975 & 0.678 & 0.766 & 0.599 & 0.647 & 0.573 & 0.621 \\
\hline & 75 & 25 & 0.615 & 0.452 & 0.260 & 0.264 & 0.208 & 0.233 & 0.202 & 0.223 \\
\hline & 75 & 50 & 0.936 & 0.705 & 0.570 & 0.382 & 0.467 & 0.312 & 0.457 & 0.300 \\
\hline & 75 & 75 & 0.985 & 0.914 & 0.757 & 0.605 & 0.663 & 0.500 & 0.639 & 0.476 \\
\hline & 75 & 100 & 0.992 & 0.973 & 0.831 & 0.738 & 0.746 & 0.611 & 0.718 & 0.582 \\
\hline & 100 & 25 & 0.682 & 0.339 & 0.270 & 0.186 & 0.212 & 0.162 & 0.197 & 0.157 \\
\hline & 100 & 50 & 0.978 & 0.771 & 0.665 & 0.463 & 0.546 & 0.398 & 0.525 & 0.381 \\
\hline & 100 & 75 & 0.997 & 0.903 & 0.833 & 0.594 & 0.734 & 0.486 & 0.707 & 0.469 \\
\hline & 100 & 100 & 0.999 & 0.978 & 0.896 & 0.771 & 0.816 & 0.661 & 0.795 & 0.634 \\
\hline
\end{tabular}

Note. $\gamma_{1}=$ Skewness and $\gamma_{2}=$ Kurtosis.

\section{Results for the Cases of Fixed Skewness}

A simulation was conducted for 20 different cases while the coefficient of skewness is taken fixed at zero. According to the simulation results, the statistical powers of both tests have been observed to increase when the coefficient of skewness is taken fixed and the coefficient of kurtosis is reduced. The greatest increase in statistical powers has occurred when the coefficient of kurtosis reduces from 0.75 to -1.00 in the case of fixed skewness. The least power increase is observed when the coefficient of kurtosis reduces from 3.75 to 3.50 in the case of fixed skewness. 
When the coefficient of skewness is reduced from 3.75 towards -1.00 in the case of fixed skewness, WW test is stronger in sample volumes $(25,25),(25,75),(25,100)$, and $(75,25)$ while KS-2 test is stronger in other sample values in terms of statistical power. The simulation results obtained due to the reduction of the skewness values in the case of fixed skewness are shown in Table 2.

Table 2

The Statistical Power Comparison of KS-2 and WW Tests When the Kurtosis Value Is Reduced in the Case of Fixed Skewness While $\sigma_{1}: \sigma_{2}=2$ in Large Sample Volumes

\begin{tabular}{|c|c|c|c|c|c|c|c|c|c|c|}
\hline \multirow{3}{*}{$\sigma_{1}: \sigma_{2}$} & \multirow{3}{*}{$n_{1}$} & \multirow{3}{*}{$n_{2}$} & \multicolumn{8}{|c|}{ Statistical power values of KS-2 and WW tests } \\
\hline & & & \multicolumn{2}{|c|}{$\begin{array}{l}\gamma_{1}=0.00 \\
\gamma_{2}=3.75\end{array}$} & \multicolumn{2}{|c|}{$\begin{array}{l}\gamma_{1}=0.00 \\
\gamma_{2}=3.50\end{array}$} & \multicolumn{2}{|c|}{$\begin{array}{l}\gamma_{1}=0.00 \\
\gamma_{2}=3.25\end{array}$} & \multicolumn{2}{|c|}{$\begin{array}{l}\gamma_{1}=0.00 \\
\gamma_{2}=3.00\end{array}$} \\
\hline & & & $\mathrm{KS}-2$ & WW & $\mathrm{KS}-2$ & WW & $\mathrm{KS}-2$ & WW & $\mathrm{KS}-2$ & WW \\
\hline \multirow{16}{*}{2} & 25 & 25 & 0.127 & 0.172 & 0.126 & 0.175 & 0.124 & 0.178 & 0.132 & 0.181 \\
\hline & 25 & 50 & 0.201 & 0.150 & 0.208 & 0.157 & 0.210 & 0.154 & 0.213 & 0.162 \\
\hline & 25 & 75 & 0.237 & 0.255 & 0.244 & 0.259 & 0.242 & 0.262 & 0.246 & 0.276 \\
\hline & 25 & 100 & 0.259 & 0.272 & 0.258 & 0.277 & 0.268 & 0.281 & 0.267 & 0.280 \\
\hline & 50 & 25 & 0.152 & 0.093 & 0.154 & 0.096 & 0.155 & 0.095 & 0.156 & 0.096 \\
\hline & 50 & 50 & 0.285 & 0.240 & 0.294 & 0.246 & 0.296 & 0.253 & 0.299 & 0.255 \\
\hline & 50 & 75 & 0.365 & 0.241 & 0.361 & 0.245 & 0.367 & 0.255 & 0.372 & 0.262 \\
\hline & 50 & 100 & 0.434 & 0.347 & 0.441 & 0.353 & 0.447 & 0.370 & 0.454 & 0.371 \\
\hline & 75 & 25 & 0.160 & 0.164 & 0.157 & 0.165 & 0.159 & 0.166 & 0.162 & 0.169 \\
\hline & 75 & 50 & 0.325 & 0.184 & 0.329 & 0.185 & 0.339 & 0.193 & 0.344 & 0.199 \\
\hline & 75 & 75 & 0.482 & 0.284 & 0.494 & 0.288 & 0.497 & 0.296 & 0.501 & 0.297 \\
\hline & 75 & 100 & 0.564 & 0.339 & 0.562 & 0.345 & 0.565 & 0.358 & 0.577 & 0.368 \\
\hline & 100 & 25 & 0.156 & 0.120 & 0.158 & 0.117 & 0.153 & 0.119 & 0.162 & 0.125 \\
\hline & 100 & 50 & 0.387 & 0.256 & 0.391 & 0.252 & 0.396 & 0.256 & 0.404 & 0.267 \\
\hline & 100 & 75 & 0.542 & 0.285 & 0.549 & 0.295 & 0.557 & 0.302 & 0.559 & 0.305 \\
\hline & 100 & 100 & 0.626 & 0.391 & 0.627 & 0.405 & 0.634 & 0.407 & 0.644 & 0.423 \\
\hline \multirow{3}{*}{$\sigma_{1}: \sigma_{2}$} & \multirow{3}{*}{$n_{1}$} & \multirow{3}{*}{$n_{2}$} & \multicolumn{8}{|c|}{ Statistical power values of KS-2 and WW tests } \\
\hline & & & \multicolumn{2}{|c|}{$\begin{array}{l}\gamma_{1}=0.00 \\
\gamma_{2}=2.75\end{array}$} & \multicolumn{2}{|c|}{$\begin{array}{l}\gamma_{1}=0.00 \\
\gamma_{2}=2.50\end{array}$} & \multicolumn{2}{|c|}{$\begin{array}{l}\gamma_{1}=0.00 \\
\gamma_{2}=2.25\end{array}$} & \multicolumn{2}{|c|}{$\begin{array}{l}\gamma_{1}=0.00 \\
\gamma_{2}=2.00\end{array}$} \\
\hline & & & KS-2 & WW & KS-2 & WW & KS-2 & WW & KS-2 & WW \\
\hline \multirow{16}{*}{2} & 25 & 25 & 0.133 & 0.189 & 0.128 & 0.188 & 0.132 & 0.187 & 0.130 & 0.190 \\
\hline & 25 & 50 & 0.212 & 0.169 & 0.212 & 0.168 & 0.218 & 0.172 & 0.219 & 0.174 \\
\hline & 25 & 75 & 0.249 & 0.280 & 0.250 & 0.288 & 0.258 & 0.295 & 0.256 & 0.307 \\
\hline & 25 & 100 & 0.268 & 0.293 & 0.274 & 0.302 & 0.274 & 0.311 & 0.276 & 0.316 \\
\hline & 50 & 25 & 0.160 & 0.100 & 0.155 & 0.094 & 0.160 & 0.102 & 0.165 & 0.105 \\
\hline & 50 & 50 & 0.304 & 0.263 & 0.303 & 0.265 & 0.308 & 0.272 & 0.310 & 0.276 \\
\hline & 50 & 75 & 0.380 & 0.265 & 0.387 & 0.277 & 0.389 & 0.282 & 0.403 & 0.294 \\
\hline & 50 & 100 & 0.460 & 0.383 & 0.461 & 0.390 & 0.472 & 0.407 & 0.479 & 0.418 \\
\hline & 75 & 25 & 0.164 & 0.171 & 0.161 & 0.172 & 0.166 & 0.178 & 0.171 & 0.184 \\
\hline & 75 & 50 & 0.354 & 0.199 & 0.352 & 0.205 & 0.361 & 0.211 & 0.370 & 0.215 \\
\hline & 75 & 75 & 0.513 & 0.311 & 0.518 & 0.314 & 0.524 & 0.320 & 0.533 & 0.334 \\
\hline & 75 & 100 & 0.585 & 0.380 & 0.588 & 0.388 & 0.599 & 0.389 & 0.614 & 0.412 \\
\hline & 100 & 25 & 0.164 & 0.125 & 0.165 & 0.123 & 0.168 & 0.125 & 0.168 & 0.132 \\
\hline & 100 & 50 & 0.407 & 0.265 & 0.418 & 0.278 & 0.415 & 0.278 & 0.430 & 0.289 \\
\hline & 100 & 75 & 0.567 & 0.314 & 0.575 & 0.313 & 0.586 & 0.324 & 0.600 & 0.338 \\
\hline & 100 & 100 & 0.654 & 0.430 & 0.656 & 0.431 & 0.669 & 0.451 & 0.678 & 0.460 \\
\hline
\end{tabular}


(Table 2 continued)

\begin{tabular}{|c|c|c|c|c|c|c|c|c|c|c|}
\hline \multirow{3}{*}{$\sigma_{1}: \sigma_{2}$} & \multirow{3}{*}{$n_{1}$} & \multirow{3}{*}{$n_{2}$} & \multicolumn{8}{|c|}{ Statistical power values of KS-2 and WW tests } \\
\hline & & & \multicolumn{2}{|c|}{$\begin{array}{l}\gamma_{1}=0.00 \\
\gamma_{2}=1.75\end{array}$} & \multicolumn{2}{|c|}{$\begin{array}{l}\gamma_{1}=0.00 \\
\gamma_{2}=1.50\end{array}$} & \multicolumn{2}{|c|}{$\begin{array}{l}\gamma_{1}=0.00 \\
\gamma_{2}=1.25\end{array}$} & \multicolumn{2}{|c|}{$\begin{array}{l}\gamma_{1}=0.00 \\
\gamma_{2}=1.00\end{array}$} \\
\hline & & & $\mathrm{KS}-2$ & WW & KS-2 & WW & KS-2 & WW & KS-2 & WW \\
\hline \multirow{16}{*}{2} & 25 & 25 & 0.134 & 0.203 & 0.144 & 0.207 & 0.139 & 0.213 & 0.140 & 0.218 \\
\hline & 25 & 50 & 0.224 & 0.189 & 0.230 & 0.189 & 0.227 & 0.193 & 0.230 & 0.202 \\
\hline & 25 & 75 & 0.266 & 0.313 & 0.271 & 0.326 & 0.276 & 0.345 & 0.276 & 0.363 \\
\hline & 25 & 100 & 0.283 & 0.330 & 0.289 & 0.342 & 0.289 & 0.349 & 0.302 & 0.374 \\
\hline & 50 & 25 & 0.166 & 0.106 & 0.165 & 0.107 & 0.167 & 0.110 & 0.173 & 0.111 \\
\hline & 50 & 50 & 0.314 & 0.287 & 0.325 & 0.303 & 0.328 & 0.303 & 0.341 & 0.321 \\
\hline & 50 & 75 & 0.405 & 0.299 & 0.418 & 0.312 & 0.420 & 0.326 & 0.431 & 0.333 \\
\hline & 50 & 100 & 0.486 & 0.427 & 0.494 & 0.447 & 0.504 & 0.465 & 0.514 & 0.489 \\
\hline & 75 & 25 & 0.174 & 0.183 & 0.175 & 0.183 & 0.178 & 0.195 & 0.181 & 0.198 \\
\hline & 75 & 50 & 0.373 & 0.225 & 0.380 & 0.231 & 0.392 & 0.240 & 0.393 & 0.244 \\
\hline & 75 & 75 & 0.543 & 0.347 & 0.551 & 0.355 & 0.563 & 0.369 & 0.577 & 0.387 \\
\hline & 75 & 100 & 0.617 & 0.412 & 0.633 & 0.433 & 0.646 & 0.446 & 0.652 & 0.469 \\
\hline & 100 & 25 & 0.170 & 0.134 & 0.177 & 0.136 & 0.176 & 0.141 & 0.184 & 0.138 \\
\hline & 100 & 50 & 0.434 & 0.296 & 0.447 & 0.305 & 0.456 & 0.306 & 0.462 & 0.317 \\
\hline & 100 & 75 & 0.602 & 0.348 & 0.605 & 0.354 & 0.630 & 0.362 & 0.640 & 0.387 \\
\hline & 100 & 100 & 0.692 & 0.479 & 0.703 & 0.495 & 0.710 & 0.503 & 0.724 & 0.522 \\
\hline \multirow{3}{*}{$\sigma_{1}: \sigma_{2}$} & \multirow{3}{*}{$n_{1}$} & \multirow{3}{*}{$n_{2}$} & \multicolumn{8}{|c|}{ Statistical power values of KS-2 and WW tests } \\
\hline & & & \multicolumn{2}{|c|}{$\begin{array}{l}\gamma_{1}=0.00 \\
\gamma_{2}=0.75\end{array}$} & \multicolumn{2}{|c|}{$\begin{array}{l}\gamma_{1}=0.00 \\
\gamma_{2}=0.50\end{array}$} & \multicolumn{2}{|c|}{$\begin{array}{l}\gamma_{1}=0.00 \\
\gamma_{2}=0.25\end{array}$} & \multicolumn{2}{|c|}{$\begin{array}{l}\gamma_{1}=0.00 \\
\gamma_{2}=0.00\end{array}$} \\
\hline & & & KS-2 & WW & $\mathrm{KS}-2$ & WW & $\mathrm{KS}-2$ & WW & KS-2 & WW \\
\hline \multirow{16}{*}{2} & 25 & 25 & 0.145 & 0.224 & 0.147 & 0.233 & 0.148 & 0.245 & 0.148 & 0.252 \\
\hline & 25 & 50 & 0.236 & 0.212 & 0.244 & 0.224 & 0.249 & 0.239 & 0.253 & 0.253 \\
\hline & 25 & 75 & 0.281 & 0.378 & 0.292 & 0.397 & 0.298 & 0.435 & 0.306 & 0.472 \\
\hline & 25 & 100 & 0.299 & 0.392 & 0.321 & 0.415 & 0.318 & 0.443 & 0.335 & 0.484 \\
\hline & 50 & 25 & 0.173 & 0.114 & 0.176 & 0.118 & 0.184 & 0.123 & 0.189 & 0.132 \\
\hline & 50 & 50 & 0.342 & 0.334 & 0.357 & 0.353 & 0.369 & 0.368 & 0.379 & 0.391 \\
\hline & 50 & 75 & 0.441 & 0.355 & 0.453 & 0.373 & 0.463 & 0.400 & 0.486 & 0.436 \\
\hline & 50 & 100 & 0.525 & 0.511 & 0.544 & 0.546 & 0.557 & 0.576 & 0.573 & 0.621 \\
\hline & 75 & 25 & 0.184 & 0.197 & 0.190 & 0.206 & 0.190 & 0.216 & 0.202 & 0.223 \\
\hline & 75 & 50 & 0.406 & 0.256 & 0.418 & 0.268 & 0.432 & 0.281 & 0.457 & 0.300 \\
\hline & 75 & 75 & 0.588 & 0.405 & 0.607 & 0.421 & 0.613 & 0.442 & 0.639 & 0.476 \\
\hline & 75 & 100 & 0.666 & 0.482 & 0.683 & 0.518 & 0.701 & 0.547 & 0.718 & 0.582 \\
\hline & 100 & 25 & 0.191 & 0.140 & 0.188 & 0.148 & 0.195 & 0.149 & 0.197 & 0.157 \\
\hline & 100 & 50 & 0.470 & 0.333 & 0.486 & 0.343 & 0.505 & 0.359 & 0.525 & 0.381 \\
\hline & 100 & 75 & 0.654 & 0.393 & 0.670 & 0.417 & 0.684 & 0.440 & 0.707 & 0.469 \\
\hline & 100 & 100 & 0.739 & 0.545 & 0.756 & 0.575 & 0.773 & 0.599 & 0.795 & 0.634 \\
\hline \multirow{3}{*}{$\sigma_{1}: \sigma_{2}$} & \multirow{3}{*}{$n_{1}$} & \multirow{3}{*}{$n_{2}$} & & & Stati & power & es of $\mathrm{KS}$ & $\mathrm{d} W \mathrm{~W}$ & & \\
\hline & & & & $\begin{array}{l}0.00 \\
-0.25\end{array}$ & & $\begin{array}{l}=0.00 \\
-0.50\end{array}$ & & $\begin{array}{l}=0.00 \\
-0.75\end{array}$ & & $\begin{array}{l}0.00 \\
-1.00 \\
\end{array}$ \\
\hline & & & $\mathrm{KS}-2$ & WW & KS-2 & WW & KS-2 & WW & $\mathrm{KS}-2$ & WW \\
\hline & 25 & 25 & 0.159 & 0.272 & 0.164 & 0.303 & 0.169 & 0.354 & 0.197 & 0.469 \\
\hline & 25 & 50 & 0.268 & 0.289 & 0.285 & 0.334 & 0.298 & 0.433 & 0.352 & 0.633 \\
\hline 2 & 25 & 75 & 0.311 & 0.522 & 0.338 & 0.603 & 0.367 & 0.733 & 0.467 & 0.896 \\
\hline & 25 & 100 & 0.342 & 0.529 & 0.360 & 0.615 & 0.395 & 0.773 & 0.480 & 0.925 \\
\hline & 50 & 25 & 0.194 & 0.142 & 0.205 & 0.155 & 0.217 & 0.181 & 0.265 & 0.273 \\
\hline
\end{tabular}


(Table 2 continued)

\begin{tabular}{|c|c|c|c|c|c|c|c|c|c|c|}
\hline \multirow{3}{*}{$\sigma_{1}: \sigma_{2}$} & \multirow{3}{*}{$n_{1}$} & \multirow{3}{*}{$n_{2}$} & \multicolumn{8}{|c|}{ Statistical power values of KS-2 and WW tests } \\
\hline & & & \multicolumn{2}{|c|}{$\begin{array}{c}\gamma_{1}=0.00 \\
\gamma_{2}=-0.25\end{array}$} & \multicolumn{2}{|c|}{$\begin{array}{c}\gamma_{1}=0.00 \\
\gamma_{2}=-0.50\end{array}$} & \multicolumn{2}{|c|}{$\begin{array}{c}\gamma_{1}=0.00 \\
\gamma_{2}=-0.75\end{array}$} & \multicolumn{2}{|c|}{$\begin{array}{c}\gamma_{1}=0.00 \\
\gamma_{2}=-1.00\end{array}$} \\
\hline & & & KS-2 & WW & KS-2 & WW & KS-2 & WW & KS-2 & WW \\
\hline & 50 & 50 & 0.396 & 0.424 & 0.419 & 0.477 & 0.459 & 0.577 & 0.581 & 0.763 \\
\hline & 50 & 75 & 0.503 & 0.479 & 0.534 & 0.551 & 0.579 & 0.682 & 0.713 & 0.879 \\
\hline & 50 & 100 & 0.594 & 0.675 & 0.629 & 0.750 & 0.688 & 0.867 & 0.816 & 0.973 \\
\hline & 75 & 25 & 0.207 & 0.233 & 0.215 & 0.251 & 0.234 & 0.290 & 0.287 & 0.386 \\
\hline & 75 & 50 & 0.469 & 0.321 & 0.499 & 0.372 & 0.553 & 0.453 & 0.707 & 0.651 \\
\hline & 75 & 75 & 0.668 & 0.524 & 0.700 & 0.587 & 0.761 & 0.701 & 0.888 & 0.889 \\
\hline & 75 & 100 & 0.742 & 0.630 & 0.780 & 0.705 & 0.833 & 0.838 & 0.936 & 0.965 \\
\hline & 100 & 25 & 0.210 & 0.167 & 0.217 & 0.184 & 0.241 & 0.210 & 0.286 & 0.282 \\
\hline & 100 & 50 & 0.548 & 0.406 & 0.576 & 0.443 & 0.641 & 0.528 & 0.806 & 0.706 \\
\hline & 100 & 75 & 0.742 & 0.512 & 0.771 & 0.569 & 0.832 & 0.675 & 0.953 & 0.873 \\
\hline & 100 & 100 & 0.819 & 0.682 & 0.850 & 0.744 & 0.898 & 0.854 & 0.979 & 0.972 \\
\hline
\end{tabular}

Note. $\gamma_{1}=$ Skewness and $\gamma_{2}=$ Kurtosis.

\section{Results and Suggestions}

According to the study, it is observed that the statistical powers of the non-parametric tests significantly increase when the coefficient of skewness is taken fixed while the coefficient of kurtosis is reduced. Similarly, a statistical power decrease is observed in non-parametric tests when the coefficient of kurtosis is taken fixed while the coefficient of skewness is reduced. In power analysis on data with fixed kurtosis, the higher statistical power may be obtained in sample distributions with higher coefficient of skewness. In the fixed kurtosis cases, the KS-2 test shows higher power performance in comparison to WW test in large sample volumes except in some sample sizes of 25 and 50. Data composed of skew distributions, call for the KS-2 test. In the fixed skewness cases, it is observed that the KS-2 test has higher statistical power in comparison to WW test in large sample volumes except in sample sizes of 25 . In this context, will the KS-2 test usually be preferred.

\section{References}

Algina, J., Olejnik, S., \& Ocanto, R. (1989). Type I error ratios and power estimates for selected two-sample tests of scale. Journal of Educational Statistics, 14(4), 373-384.

Balakrishnan, N., \& Nevzorov, V. B. (2003). A primer on statistical distributions. Hoboken, New Jersey: John Wiley \& Sons, Inc.. Conover. W. J. (1999). Practical nonparametric statistics (3th ed.). New York: John Wiley \& Sons, Inc..

Daniel, W. W. (1990). Applied nonparametric statistics (2th ed.). Boston: PWS-Kent Publishing Company.

Higgins, J. J. (2004). Introduction to modern nonparametric statistics. Pacific Grove: Thomson Learning, Inc..

Joanest, D. N., \& Gill, C. A. (1998). Comparing measures of sample skewness and kurtosis. The Statistician, 47(1), $183-189$.

Magel, R. C., \& Wibowo, S. H. (1997). Comparing the powers of the Wald-Wolfowitz and Kolmogorov-Smirnov tests. Biometrical Journal, 39(6), 665-675.

Marascuilo, L. A., \& McSweeney, M. (1977). Nonparametric and distribution-free methods for social science. Monterey, C.A.: Brooks/Cole Publishing Company.

Mehta, C. R., \& Patel, N. R. (1996). SPSS exact tests 7.0 for Windows. Chicago: SPSS Inc..

Mooney, C. Z. (1997). Monte Carlo simulation. Thousand Oaks, C.A.: SAGE Publications, Inc..

Roese, J. H. (2011). Wald-Wolfowitz runs test. Hetrieved from http://www.lssu.edu/faculty/jroese/recipes/S2V1/wald_wolfowitz.html

Sahai, H., \& Khurshid, A. (2002). Pocket dictionary of statistics. Irwin: McGraw-Hill.

Sheskin, D. J. (2000). Handbook of parametric and nonparametric statistical procedures. Baca Raton: Chapman \& Hall/CRC.

Sprent, P., \& Smeeton, N. C. (2001). Applied nonparametric statistical methods (3rd ed.). Florida: Chapman \& Hall/CRC. 